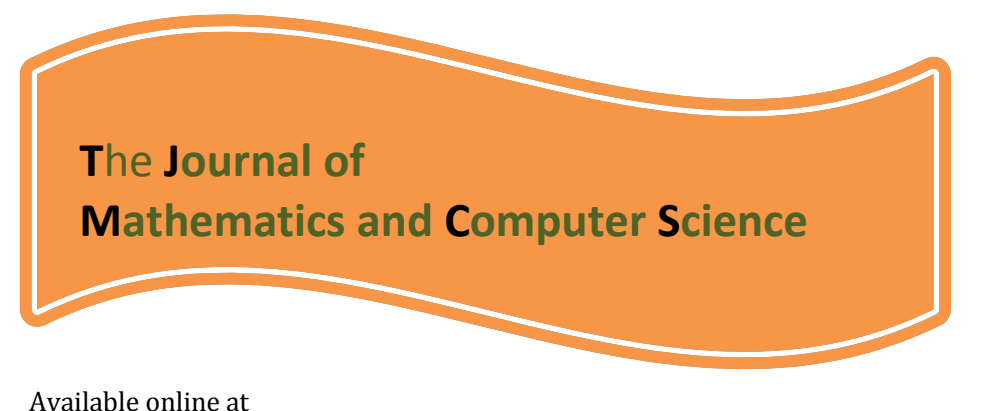

\title{
http://www.TJMCS.com
}

The Journal of Mathematics and Computer Science Vol. 4 No.3 (2012) 380 - 385

\section{Ergodicity of Fuzzy Markov Chains Based on Simulation Using Halton Sequences}

\author{
Behrouz Fathi Vajargah ${ }^{1}$, Maryam Gharehdaghi ${ }^{2}$ \\ ${ }^{1}$ Department of Statistics, University of Guilan, Rasht, Iran, \\ fathi@guilan.ac.ir \\ ${ }^{2}$ Department of Statistics, University of Guilan, Rasht, Iran, \\ gharehdaghi.m@gmail.com
}

Received: February 2012, Revised: May 2012

Online Publication: July 2012

\begin{abstract}
We first introduce fuzzy finite Markov chains and present some of their fundamental properties based on possibility theory. We also bring in a way to convert fuzzy Markov chains to classic Markov chains. In addition, we simulate fuzzy Markov chain using different sizes. It is observed that the most of fuzzy Markov chains not only do have an ergodic behavior, but also they are periodic. Finally, using Halton quasi-random sequence we generate some fuzzy Markov chains which compared to the ones generated by the RAND function of MATLAB. Therefore, we improve the periodicity behavior of fuzzy Markov chains.
\end{abstract}

Keywords: Fuzzy Markov Chains, Stationary Distribution, Ergodicity, Simulation, Halton QuasiRandom Sequence.

\section{Introduction}

The main motivation of this paper is to begin the expansion of fuzzy Markov chains based on possibilities. A reason is that one might prefer subjective possibilities over subjective probabilities to model the uncertainties.

Markov chains are important tools for solving practical problems. Their applications for many problems with successful results are well known. The fuzzy Markov chains approaches are given by Avrachenkov and Sanchez in [2] and also [8] by using the max-min operator on fuzzy sets to find their fuzzy stationary behavior. The theoretical distinction between the fuzzy Markov chains and classic Markov chains lies in the functional form of their max-min operators.

This paper is divided into five sections. Section 1 is an introductory section. In section 2 some concepts of fuzzy Markov chains and their properties are defined [1] and [2]. In section 3, a linear transformation of the fuzzy Markov chains into a classic Markov chains is given [7]. In section 4 of

\footnotetext{
$1{ }^{*}$ Corresponding author: Associate Professor in Statistics

${ }^{2}$ PhD Student in Applied Mathematics
} 
this paper, we employ Halton quasi-random sequence to generate membership functions of fuzzy Markov chains. At last in section 5 we simulate and compare the generated fuzzy Markov chains obtain from both Holton method and the MATLAB RAND function, and finally the section 6 presents the concluding remarks.

\section{Basic definitions of fuzzy Markov chains and their properties}

The definition of a fuzzy Markov chain is based on a squared relational matrix represents the possibility that a discrete state at instant $t$ becomes into any state at next instant $t+1$ as follows:

$$
\bar{P}\left(X^{(t)}=s \mid X^{(t-1)}\right)=x^{(t-1)} .
$$

Here, $\bar{P}\left(X^{(t)}\right)$ is a fuzzy distribution of the process characterized by a membership function. In this paper, we use the basic definitions about fuzzy set given by J. Buckley in [3] and fuzzy Markov chains given by Avrachenkov and Sanchez in [2].

Definition 2.1. Let $S=\{1,2, \ldots, n\}$. A finite fuzzy set for a fuzzy distribution on $S$ is defined by a mapping $x$ from $S$ to $[0,1]$ represented by a vector $x=\left\{x_{1}, x_{2}, \ldots, x_{n}\right\}$, with $0 \leq x_{i} \leq 1, i \in S$.

In this definition, $x_{i}$ is the membership degree that a state $i$ has regarding a fuzzy set $S, i \in S$ with cardinality $m, \mathcal{C}(S)=m$. All relations and compositions are defined by fuzzy sets theory since are useful tools to find a fuzzy stationary distribution.

Now, a fuzzy relational matrix $\bar{P}$ is defined in a metric space $S \times S$ by a matrix $\left\{\bar{p}_{i j}\right\}_{i, j=1}^{m}$ with $0 \leq \bar{p}_{i j} \leq 1, i, j \in S$. The complete set of all fuzzy sets is denoted by $\mathcal{F}(S)$ where $\mathcal{C}(S)=m$.

We note that it does not need the addition of elements of each row of the matrix $\bar{P}$ be equal to one [3].

This fuzzy matrix $\bar{P}$ allows defining all relations among the $m$ states of the fuzzy Markov chain at each time instant $t$, as follows.

Definition 2.2. At each instant $t, t=1,2, \ldots, n$, the state of system is described by the fuzzy set $x^{(t)} \in \mathcal{F}(S)$. The transition law of a fuzzy Markov chain is given by the fuzzy relational matrix $\bar{P}$ at instant $t, t=1,2, \ldots, n$, as follows:

$$
\begin{aligned}
& x_{j}^{(t+1)}=\max _{i \in S}\left\{x_{j}^{(t)} \wedge \bar{p}_{i j}\right\}, j \in S, \\
& x^{(t+1)}=x^{(t) \circ} \bar{P},
\end{aligned}
$$

where $i$ and $j, i, j=1,2, \ldots, m$ are the initial and final states of the transition and $x^{(0)}$ is the initial distribution. Also,

$$
\begin{aligned}
& \bar{p}_{i j}^{t}=\max _{k \in S}\left\{\bar{p}_{i k} \wedge \bar{p}_{k j}^{t-1}\right\}, i, j \in S, \\
& \bar{P}^{t}=\bar{P}^{\circ} \bar{P}^{t-1} .
\end{aligned}
$$

Thomason in [9] showed that the powers of a fuzzy matrix are stable if it is used the max-min operator. More information about powers of a fuzzy matrix are, see in [2], [5], [4]. Now, a stationary distribution of a fuzzy matrix defined as follow [7].

Definition 2.3 (Stationary distribution). Let the powers of the fuzzy transition matrix $\bar{P}$ converge in $\tau$ steps to a non-periodic solution, and then the associated fuzzy Markov chain is called aperiodic fuzzy Markov chain and $\bar{P}^{*}=\bar{P}^{\tau}$ is its stationary fuzzy transition matrix. 
Definition 2.4 (Strong Ergodicity and Week Periodicity). A fuzzy Markov chain is called strong Ergodic if it is aperiodic and its stationary transition matrix has identical rows.

A fuzzy Markov chain is called weakly Ergodic if it is aperiodic and its stationary transition matrix is stable with no identical rows.

Now, if the stationary distribution of $\bar{P}$ is given by $\bar{P}^{*}=\bar{P}^{\tau}$ where $\lim _{n \rightarrow \tau} \bar{P}^{n}=\bar{P}^{*}$, then $\bar{P}$ becomes an idempotent matrix. Sanchez defined its stationary distribution by its Eigen fuzzy set, see in [8].

\section{Converting a fuzzy Markov chain to a classic Markov chain}

The scalar cardinality and the cumulative membership function of a fuzzy set is used to define a conversion of fuzzy Markov chain into a classic Markov chain [7].

Definition 3.1 (Scalar cardinality of a fuzzy set). The well known scalar cardinality of a fuzzy set namely $|S|$ is a measure of amount and represents the total size of the membership function of $S\left(\mu_{S}\right)$ as follows:

$$
\Lambda_{i}=\left|S_{i}\right|=\sum_{j=1}^{m} \mu_{S_{i j}}, i \in m,
$$

Where $\Lambda$ a diagonal squared matrix is whose components are the scaler cardinality of each set $\left|S_{i}\right|$ by row, denoted by $\Lambda_{i}, i \in m$.

Definition 3.2 (Cumulative membership function). The cumulative membership function is defined as:

$$
\psi_{S_{i}}=\sum_{j=1}^{x} \mu_{S_{i j}}, i \in m \text {. }
$$

Note that in the probabilistic case $F(\infty)=1$ while in the possibilistic case $1<\psi(\infty)<\Lambda$. also $\mu_{S}(\infty)>1$ is an important issue to be solved. This is an interpretation problem since the definition of a normalized fuzzy set determines that $\max _{x \in S} \mu_{S}(x)=1$ and the above definition does not have this property. To solve it, an easy way to normalize $\psi_{S}(x)$ is dividing it by $\Lambda$, obtaining the following definition:

$$
\psi_{S_{i}}=\frac{1}{\Lambda_{i}} \sum_{j=1}^{x} \mu_{S_{i j}}, i \in m \text {. }
$$

Remark 1 (Relation between $\psi_{S}(x)$ and $\left.|S|\right)$. Recall that $\psi_{S}(\infty)=\Lambda$.

Theorem 3.1. Let $\bar{P}$ a fuzzy Markov chain with elements $\quad \bar{p}_{i j}=\mu_{S_{i j}}$, then $\bar{P}$ can be converted to a classic Markov chain namely $P$, using the following linear transformation:

$$
P=\Lambda^{-1} \bar{P} \text {. }
$$

Proof. First, the scaler cardinality of $S_{i}$ defined as (6), so, $\Lambda, \Lambda^{-1}$ and $\bar{P}$ are defined as:

$$
\Lambda=\left(\begin{array}{cccc}
\Lambda_{1} & & & \\
& \Lambda_{2} & & \\
& & \ddots & \\
& & & \Lambda_{m}
\end{array}\right) ; \Lambda^{-1}=\left(\begin{array}{cccc}
\Lambda_{1}^{-1} & & & \\
& \Lambda_{2}^{-1} & & \\
& & \ddots & \\
& & & \Lambda_{m}^{-1}
\end{array}\right) ; \bar{P}=\left(\begin{array}{cccc}
\mu_{S_{11}} & \mu_{S_{12}} & \ldots & \mu_{S_{1 m}} \\
\mu_{S_{21}} & \mu_{S_{22}} & \ldots & \mu_{S_{2 m}} \\
\vdots & \vdots & \ddots & \vdots \\
\mu_{S_{m 1}} & \mu_{S_{m 2}} & \ldots & \mu_{S_{m m}}
\end{array}\right) \text {. }
$$


Finally we have

$$
P=\left(\begin{array}{cccc}
\Lambda_{1}^{-1} & & & \\
& \Lambda_{2}^{-1} & & \\
& & \ddots & \\
& & & \Lambda_{m}^{-1}
\end{array}\right) *\left(\begin{array}{cccc}
\mu_{S_{11}} & \mu_{S_{12}} & \ldots & \mu_{S_{1 m}} \\
\mu_{S_{21}} & \mu_{S_{22}} & \ldots & \mu_{S_{2 m}} \\
\vdots & \vdots & \ddots & \vdots \\
\mu_{S_{m 1}} & \mu_{S_{m 2}} & \ldots & \mu_{S_{m m}}
\end{array}\right),
$$

which is $P=\Lambda^{-1} \bar{P}$. Now, all their components result in the following matrix:

$$
P=\left(\begin{array}{cccc}
\frac{\mu_{S_{11}}}{\Lambda_{1}} & \frac{\mu_{S_{12}}}{\Lambda_{1}} & \ldots & \frac{\mu_{S_{1 m}}}{\Lambda_{1}} \\
\frac{\mu_{S_{21}}}{\Lambda_{2}} & \frac{\mu_{S_{22}}}{\Lambda_{2}} & \ldots & \frac{\mu_{S_{2 m}}}{\Lambda_{2}} \\
\vdots & \vdots & \ddots & \vdots \\
\frac{\mu_{S_{m 1}}}{\Lambda_{m}} & \frac{\mu_{S_{m 2}}}{\Lambda_{m}} & \ldots & \frac{\mu_{S_{m m}}}{\Lambda_{m}}
\end{array}\right) .
$$

This transformation obtains a matrix $P$ whose elements satisfy all basic properties of a stochastic classic distribution function, i.e.

$$
\begin{aligned}
& \text { i) } 0 \leq p\left(x_{i j}\right) \leq 1 \\
& \text { ii) } \sum_{j \in S}^{\infty} p\left(x_{i j}\right)=1, i \in S \\
& \text { iii) } P\left(X \leq x_{i j}\right)=\sum_{i \leq S} p\left(x_{i j}\right)=F(x), i \in S
\end{aligned}
$$

In this way, it is possible to show that all elements of $P$ obtained from $\bar{P}$ by using $P=\Lambda^{-1} \bar{P}$ conforms a classic distribution function; agree to the Markovian property of a stochastic transition matrix. To that effect, their properties are shown next.

The property presented in $i$ refers to the domain of $p_{i j}$, and as $\mu_{S_{i j}}<\Lambda_{i}, i, j \in m$, then it asserts that $\frac{\mu_{S_{i j}}}{\Lambda_{i}}<1, i, j \in m$.

\section{Halton Sequence for generating $\mu_{S_{i j}}$}

In 1960, a Halton quasi-random sequence is introduced by reversing the digits in the representation of

some sequence of integers in a given base. Although this can be done somewhat arbitrarily, astraightforward way of forming a d-dimensional Halton sequence $\mu_{1}, \mu_{2}, \ldots$, where $\mu_{i}=\left(\mu_{S_{i 1}}, \mu_{S_{i 2}}, \ldots, \mu_{S_{i d}}\right)$, is first to choose $d$ bases $b_{1}, b_{2}, \ldots, b_{d}$, perhaps the first $d$ primes. The $j^{\text {th }}$ base will be used to form the $j^{\text {th }}$ component of eachvector in the sequence [6]. Then, begin with some integer $\ell$ and

1. choosing $t_{\ell j}$ suitably large, represent $\ell$ in each base:

$$
\ell=\sum_{k=0}^{t_{f_{j}}} a_{j k}(\ell) b_{j}^{k}, j=1,2, \ldots, d .
$$

2. Form

$$
\mu_{S_{i j}}=\sum_{k=0}^{t_{\ell_{j}}} a_{j k}(\ell) b_{j}^{k-t_{\ell_{j}}-1}, j=1,2, \ldots, d .
$$

3. Set $\ell=\ell+1$ and repeat. 


\section{Simulation}

We present a simulation on fuzzy Markov chains to identify some characteristics about their behavior based on matrix analysis.

Algorithm: Now, using the linear operation given in section 3, we simulate the fuzzy Markov chains.

Size of the fuzzy Markov chains: The size of $\bar{P}$ denoted by $m, m=\{5,10,50,100\}$.

Random number generator: $\}$ All the entries $\left\{\bar{p}_{i j}\right\}=\left\{\mu_{i j}\right\}$ of the matrix $\bar{P}$ are obtained by using the RAND function of MATLAB (Table 1) and Halton quasi-random sequence (Table 2).

Number of runs: 100 runs are simulated for per each size of $\bar{P}$.

Table 1. Total number of strong ergodicity, weak ergodicity and periodic chains using the RAND function of MATLAB.

\begin{tabular}{lcccc}
\hline Size & Strong ergodicity & Weak ergodicity & Periodic & Total \\
\hline $\mathbf{m}=\mathbf{5}$ & 12 & 32 & 56 & 100 \\
$\mathbf{m}=\mathbf{1 0}$ & 3 & 11 & 86 & 100 \\
$\mathbf{m}=\mathbf{5 0}$ & 1 & 3 & 96 & 100 \\
$\mathbf{m}=\mathbf{1 0 0}$ & - & 1 & 99 & 100 \\
\hline
\end{tabular}

Table 2. Total number of strong ergodicity, weak ergodicity and periodic chains using Halton quasi-random sequence

\begin{tabular}{lcccc}
\hline Size & Strong ergodicity & Weak ergodicity & Periodic & Total \\
\hline $\mathbf{m}=\mathbf{5}$ & 26 & 39 & 35 & 100 \\
$\mathbf{m}=\mathbf{1 0}$ & 14 & 21 & 65 & 100 \\
$\mathbf{m}=\mathbf{5 0}$ & 9 & 14 & 77 & 100 \\
$\mathbf{m}=\mathbf{1 0 0}$ & 3 & 6 & 91 & 100 \\
\hline
\end{tabular}

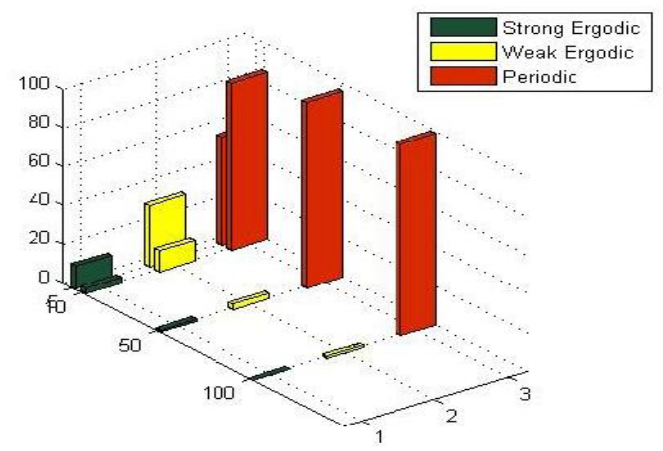

Figure 1. Strong ergodicity, weak ergodicity and periodic chains using the RAND function of MATLAB.

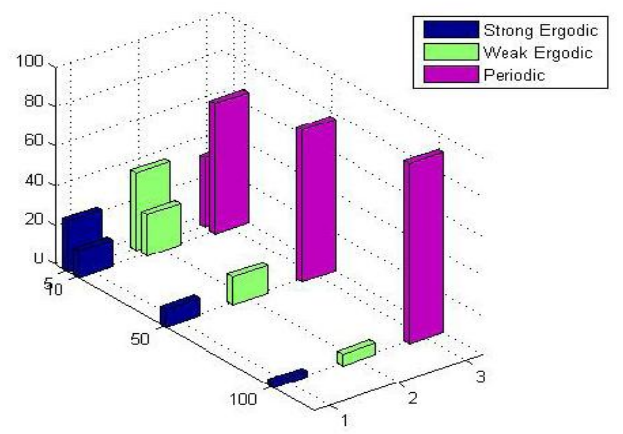

Figure 2. Strong ergodicity, weak ergodicity and periodic chains using Halton quasi-random sequence. 
The Table 1 and Table 2 as well as Figure 1 and Figure 2 show the total number of fuzzy Markov chains which has either a strong ergodic, weak ergodic or periodic behavior per each size of $P$.

\section{Conclusion}

Based on our presented results and former related results we conclude that the fuzzy Markov chains are usually periodic, and the fuzzy Makov chains generated by Halton method are more efficient in terms of periodicity than those generated by RAND function.

We shall deal with stationary and ergodic fuzzy Markov chains. We propose some algorithms to make such periodic fuzzy Markov chains ergodic.

\section{References}

[1] Araiza, R., Xiang, G., Kosheleva, O., Skulj, D.: Under interval and fuzzy uncertainty, symmetric Markov chains are more difficult to predict. Annual Meeting of the North American Fuzzy Information Processing Society (NAFIPS), IEEE, Los Alamitos, 526-531 (2007).

[2] Avrachenkov, K.E., Sanchez, E.: Fuzzy Markov chains. IPMU, Spain, 1851-1856 (2000).

[3] Buckley, J.J.: Fuzzy Probability and Statistics. F. Verlag, Ed. Springer-Verlag, (2004).

[4] Gavalec, M.: Computing orbit period in max-min algebra. Discrete Applied Mathematics 100, 4965 (2000).

[5] Gavalec, M.: Periods of special fuzzy matrices. Tatra Mountains Mathematical publication 16, 4760(1999).

[6] Gentle, J.E.: Random Number Generation and Monte Carlo Methods. Springer, (2005).

[7] Kalenatic, D., Figueroa-Garcia, J., Lopez, C.A.: Scalarization of Type-1 Fuzzy Markov Chains. LNCS, Springer-Verlag, 110-117 (2010).

[8] Sanchez, E.: Eigen fuzzy sets and fuzzy relations. Journal of mathematical analysis and applications 81, 399-421 (1981).

[9] Thomason, M.: Convergence of powers of a fuzzy matrix. Journal of mathematical analysis and applications 57, 476-480 (1977). 